\title{
Stage III Jejunal Neuroendocrine Tumor AJCC v8
}

National Cancer Institute

\section{Source}

National Cancer Institute. Stage III Jejunal Neuroendocrine Tumor A/CC v8. NCI

Thesaurus. Code C135122.

Stage III includes: (T1, N1, N2, M0); (T2, N1, N2, M0); (T3, N1, N2, M0); (T4, N0, M0); (T4, N1, N2, M0). T1: Tumor invading lamina propria or submucosa and measuring $1 \mathrm{~cm}$ or less in size. T2: Tumor invading muscularis propria or measuring more than $1 \mathrm{~cm}$ in size. T3: Tumor invading through the muscularis propria into subserosal tissue without penetration of overlying serosa. T4: Tumor invading visceral peritoneum (serosa) or other organs or adjacent structures. N0: No regional lymph node metastasis. N1: Regional lymph node metastasis in less than 12 nodes. N2: Large mesenteric masses (larger than $2 \mathrm{~cm}$ ) and/or extensive nodal deposits (12 or greater), especially those that encase the superior mesenteric vessels. M0: No distant metastasis. (AJCC 8th ed.) 